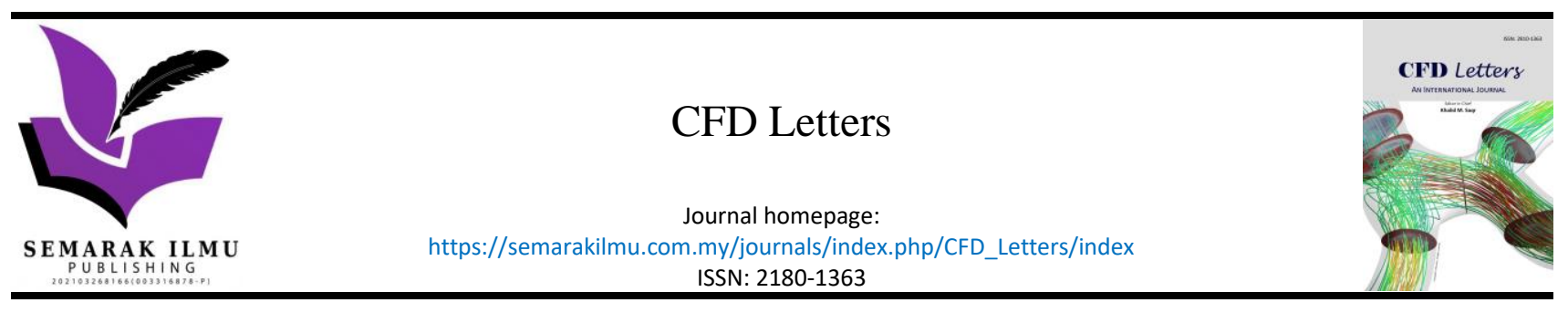

\title{
Rayleigh-Benard Convection in Nanofluids Layer saturated in a Rotating Anisotropic Porous Medium with Feedback Control and Internal Heat Source
}

\author{
Izzati Khalidah Khalid ${ }^{1,}$, , Nor Fadzillah Mohd Mokhtar ${ }^{2,3}$, Zarina Bibi Ibrahim ${ }^{3}$ \\ Faculty of Computer and Mathematical Sciences, Universiti Teknologi MARA, 40450 Shah Alam, Selangor, Malaysia \\ Laboratory of Computational Sciences and Mathematical Physics, Institute for Mathematical Research (INSPEM), Universiti Putra Malaysia, \\ 43400 Serdang, Selangor, Malaysia \\ 3 Department of Mathematics, Faculty of Science, Universiti Putra Malaysia, 43400 Serdang, Selangor, Malaysia
}

\section{ARTICLE INFO ABSTRACT}

\section{Article history:}

Received 16 August 2021

Received in revised form 17 October 2021

Accepted 9 December 18 October 2021

Available online 11 November 2021

\section{Keywords:}

Feedback Control; Rotation; Nanofluids; Internal Heat Source; Porous Medium

\begin{abstract}
Control strategy on Rayleigh-Benard convection in rotating nanofluids saturated in anisotropic porous layer heated from below is studied in the presence of uniformly internal heat source for rigid-rigid, free-free, and lower-rigid and upper-free boundaries. Feedback control strategy with an array of sensors situated at the top plate and actuators located at the bottom plate of the nanofluids layer are considered in this study. Linear stability analysis based on normal mode technique has been performed, the eigenvalue problem is obtained numerically by implementing the Galerkin method and computed by using Maple software. Model employed for the nanofluids includes the mechanisms of Brownian motion and thermophoresis. The problem of the onset of convective rolls instabilities in a horizontal porous layer with isothermal boundaries at unequal temperatures known as Horton-Roger-Lapwood model based on the Darcy model for the fluids flow is used. The influences of internal heat source's strength, modified diffusivity ratio, nanoparticles concentration DarcyRayleigh number and nanofluids Lewis number are found to advance the onset of convection, meanwhile the mechanical anisotropy parameter, thermal anisotropy parameter, porosity, rotation, and controller effects are to slow down the process of convective instability. No visible observation on the modified particle density increment and rigid-rigid boundaries are the most stable system compared to free-free and rigid-free boundaries.
\end{abstract}

\section{Introduction}

Nanofluids are colloidal suspensions of nanoparticles $(1-100 \mathrm{~nm})$ in a base fluid and the term for nanofluids which define as fluids with nanosized particles $(1-10 \mathrm{~nm})$ suspended stably in them was proposed by Choi [1]. The base fluids alone have rather low thermal conductivities and by suspending particles in a base liquid helps to improve the thermal conductivity. This is mainly thought to be due to Brownian motion preventing gravity settling and agglomeration of particles, resulting in a much

\footnotetext{
* Corresponding author.

E-mail address: izzatikk@uitm.edu.my (Izzati Khalidah Khalid)
} 
more stable, suspended fluid. These new-formation heat transfer fluids offered a new possibilities motivation to improve the performance of heat transfer compared to regular fluids. The enhancement depends on several aspects as reported by Menni et al., [2] such as shape and size of the particles, volume fraction of particles, and thermal properties of solid and liquid. The analysis of the literature dealing with recent developments in focusing on the convective heat transfer by using nanofluids can be extracted in the review articles [3-7]. Tzou [8, 9] studied buoyancy driven convection in a horizontal nanofluid layer heated from below on the basis of the transport equations promoted by Buongiorno [10], who pointed the absolute velocity of nanoparticles as the sum of the base fluid velocity and a relative velocity. Kim et al., [11] introduced a new factor which determines the effect of nanoparticles addition on a base fluid and analyzed the convective instability driven by thermal gradient as well as heat transfer characteristics of nanofluids. Nield and Kuznetsov [12] studied the onset of convection in nanofluids layer of finite depth. Linear stability analysis of RayleighBenard convection in nanofluids layer for free-free boundaries was performed by Yadav et al., [13]. Hadad et al., [14] reported that thermophoresis and Brownian motion mechanisms are significant in thermal enhancement of the natural convection in nanofluids layer. A review on advances of nanofluids in heat exchangers, that is, heat pipe and plate-fin heat exchangers has been reviewed by Sharma et al., [15]. Modified model for Binary Nanofluid convection with initial constant nanoparticle volume fraction has been investigated by Menni et al., [16]. Gupta et al., [17] employed the Casson nanofluid convection in an internally heated layer. Recently, Gupta et al., [18] studied the doublediffusive instability of Casson nanofluids with numerical investigations for blood-based fluid. Meanwhile, Aliouane et al., [19] investigated the flow and thermal fields in square enclosures: Rayleigh-Benard's instabilities of nanofluids. The effect of turbulent flow and convective heat transfer of $\mathrm{Al}_{2} \mathrm{O}_{3}$-water nanofluids in a circular tube for numerical study has been examined by Mahammedi et al., [20]. For partially porous cavity in the presence of nanofluids laminar naturel convection has been studied by Douha et al., [21]. Numerical research on the magnetic Ni nanofluid flowing in a tube, developing turbulent flows under constant heat flux conditions has been reported by Abdelkader et al., [22].

Thermal instability induced by a uniform internally heat source arising in horizontal layer of fluids has attracted investigators attention for many decades ago. Sparrow et al., [23] studied analytically the problem of thermal instability of an internally heated fluid as well as heated from below, and showed that the effect of internal heat source destabilized the system. Char and Chiang [24] proved that Benard-Marangoni convection critically induced with increasing internal heat source rate the system is prone to instability. Nield and Kuznetsov [25] investigated the thermal instability in a porous medium layer saturated by a nanofluids. Nanjundappa et al., [26] studied the effect of uniform internal heat source on Brinkman-Benard convection in a ferrofluid saturated porous layer. The effect of internal heat source on the onset of Darcy-Brinkman convection in a porous layer saturated by a nanofluid is investigated by Yadav et al., [27]. Shivakumara and Dhananjaya [28] investigated the penetrative Brinkman convection in an anisotropic porous layer saturated by a nanofluid layer. Chand et al., [29] studied the effects of variable gravity on thermal instability in a horizontal layer of a nanofluid saturating an anisotropic Darcy porous medium. These researchers [30-33] studied the anisotropic porous medium with various types of fluids such as binary fluid, fluid, ferrofluids and various effects of nonlinear temperature profile, magnetic field with rotation, internal heating and variable gravity.

The use of feedback control in stabilizing the thermal convection was investigated by Wang et al., [34] and they managed to inhibit the chaotic behavior in the fluid layer by applying proportional control in a thermal convection loop. Tang and Bau [35] showed that, with the use of feedback controller, the critical Rayleigh number for the onset of convection can be significantly increased. The 
similar control strategy can be used to modify the flow patterns of surface-tension driven flows as reported by Bau [36]. Hashim and Siri [37] have attempted to include the stabilizing effect of feedback control on steady and oscillatory convection due to surface-tension. The effects of feedback control on chaotic convection in fluid-saturated porous media has been investigated theoretically using dynamical systems approach by Roslan et al., [38]. They showed that the suppression of chaotic convection in possible via feedback control. The investigation on the effect of feedback control in small and moderate Vadasz number chaotic convection in porous media was extended by Mahmud and Hashim [39] in the presence of non-Boussinesq effect using Galerkin truncated approximation technique.

Thermal convection in a rotating fluid saturated porous layer heated from below has been the subject of experimental and theoretical interest by many researchers. Vadasz [40] investigated the convective instability for the effect of rotating porous layer and found that it stabilized the system. The Coriolis force which is due to rotation in the system has an important effect of convective instability, where its effect helps to delay the onset of convection. Meanwhile, Govender [41] added the stabilizing effect of Coriolis force in anisotropic porous layer subjected to gravity. In 2020, Khalid et al., [42] investigated the stabilizing effect of control strategy on Rayleigh-Benard convection in Maxwell nanofluids layer saturated in a rotating porous medium with feedback control subjected to viscosity and thermal conductivity variations. Sharma and Gupta [43] studied the onset of doublediffusive convection in a rotating binary nanofluid layer saturated by a porous medium using DarcyBrinkman model. Later, Sharma et al., [44] investigated the convection in a rotating binary nanofluid layer in porous medium using Darcy-Brinkman model.

The motivation of the present paper is to study the effect of controller on the onset of convection in a horizontal layer of an anisotropic porous medium (the Horton-Rogers-Lapwood problem as discussed by Nield and Kuznetsov [25]) saturated by a nanofluid heated from below with internal heat source, since there is no research reported on this investigation from the previous researchers. The study finds relevance in many applications particularly in manufacturing processes in industry. It is imperative to note that the internal heat source changes the temperature distribution significantly in the nanofluids which eventually alters the particles deposition rate in nuclear reactors, electronic chips and semiconductor wafers as mentioned by Aliouane et al., [19]. The eigenvalue problem is extracted numerically using the Galerkin method for different types of velocity boundary conditions and solved by using Maple software. The study undertaken is more general in the sense that the results for the Darcy porous medium as well as for a fluid layer can be delineated as particular cases from the present study.

\section{Methodology}

Cartesian coordinates $(x, y, z)$ are used, where the $z$-axis points vertically upward. Consider a horizontal layer of a rotating incompressible nanofluids saturated anisotropic porous layer (the Horton-Rogers-Lapwood problem) of thickness $L$ confined between the planes $z^{*} \in[0, L]$ and subjected to internal heat source $Q_{0}^{*}$ and feedback control is heated from below as shown in Figure 1 . The nanofluids layer rotates about the vertical axis at a constant angular velocity, $\Omega^{*}=(0,0, \Omega)$. The nanoparticles are suspended in nanofluids using either surfactant or surface charge technology. This prevents particles from agglomeration and deposition on the porous matrix. For simplicity, Darcy's Law is assumed to hold and the Oberbeck-Boussinesq approximation is employed. Homogeneity and local thermal equilibrium in the porous medium are assumed. Following Nield and Kuznetsov [25] and Yadav et al., [27], the governing equations under the Oberbeck-Boussinesq approximation are 
the conservation of mass, momentum, thermal energy and nanoparticles, and they are respectively given by

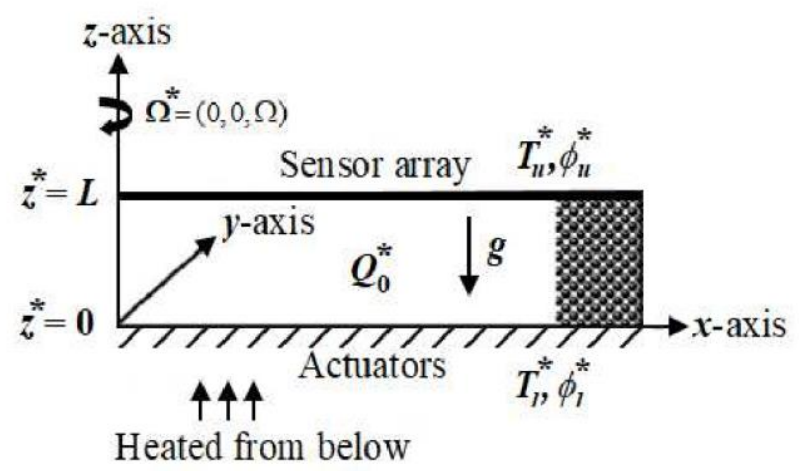

Fig. 1. Physical configuration and coordinate system

$\nabla^{*} \cdot \boldsymbol{u}_{D}^{*}=0$

$-\nabla^{*} p^{*}-\mu \frac{\boldsymbol{u}_{D}^{*}}{\tilde{K}}+\boldsymbol{g}\left\{\phi^{*} \rho_{p}+\left(1-\phi^{*}\right) \rho_{f}\left[1-\alpha_{T}\left(T^{*}-T_{u}^{*}\right)\right]\right\}-\frac{2 \rho_{f}}{\varepsilon}\left(\boldsymbol{\Omega}^{*} \times \boldsymbol{u}_{D}^{*}\right)=0$,

$(\rho c)_{m} \frac{\partial T^{*}}{\partial t^{*}}+(\rho c)_{f}\left(\boldsymbol{u}_{D}^{*} \cdot \nabla^{*}\right) T^{*}-\tilde{\kappa}_{m} \nabla^{* 2} T^{*}-Q_{0}^{*}-\varepsilon(\rho c)_{p}\left[D_{B} \nabla^{*} \phi^{*} \cdot \nabla^{*} T^{*}+\left(\frac{D_{T}}{T_{u}^{*}}\right) \nabla^{*} T^{*} \cdot \nabla^{*} T^{*}\right]=0$

$\frac{\partial \phi^{*}}{\partial t^{*}}+\frac{1}{\varepsilon}\left(\boldsymbol{u}_{D}^{*} \cdot \nabla^{*}\right) \phi^{*}-D_{B} \nabla^{* 2} \phi^{*}-\frac{D_{T}}{T_{u}^{*}} \nabla^{* 2} T^{*}=0$,

where $\boldsymbol{u}_{D}^{*}=(u, v, w)$ is the Darcy velocity, $\rho_{f}$ is the density of the base fluid, $\rho_{p}$ is the nanoparticle mass density, $t^{*}$ is the time, $p^{*}$ is the pressure, $\mu$ is the viscosity, $\tilde{K}$ is the permeability of the porous medium, $\varepsilon$ is the porosity of the porous medium, $g$ is the gravitational force, $\phi^{*}$ is the nanoparticle volume fraction, $\alpha_{T}$ is the thermal volumetric coefficient, $T^{*}$ is the temperature, $(\rho c)_{m}$ is the effective heat capacity, $c$ is the specific heat, $c_{p}$ is the specific heat of the nanoparticles, $Q_{0}^{*}$ is the uniform internal heat source, $\tilde{\kappa}_{m}$ is the effective thermal conductivity of the porous medium saturated by the nanofluid, $D_{B}$ is the Brownian diffusion coefficient and $D_{T}$ is the thermophoretic diffusion coefficient. The permeability and thermal conductivity tensors are defined as

$$
\begin{aligned}
& \frac{1}{\tilde{K}}=\frac{1}{\tilde{K}_{H}}(\hat{i i}+\hat{j j})+\frac{1}{\tilde{K}_{V}} \hat{k} \hat{k}, \\
& \tilde{\kappa}_{m}=\tilde{\kappa}_{m H}(\hat{i i}+\hat{j} \hat{j})+\tilde{\kappa}_{m V} \hat{k} \hat{k},
\end{aligned}
$$


where $\tilde{K}_{H}$ is the permeability and $\tilde{\kappa}_{m H}$ is the thermal conductivity in the horizontal $\hat{i}$ and $\hat{j}$ directions, while $\tilde{K}_{V}$ and $\tilde{\kappa}_{m V}$ are the corresponding values in the vertical $\hat{k}$ direction. It may be noted that horizontal mechanical and thermal isotropy has been assumed.

It is assumed that the temperature and volumetric fraction of the nanoparticles are constant on the boundaries. Thus, the boundary conditions are

$$
\begin{aligned}
& w^{*}=0, \frac{\partial w^{*}}{\partial z^{*}}+\alpha_{1} L \frac{\partial^{2} w^{*}}{\partial z^{* 2}}=0, T^{*}=T_{0}^{*}, \phi^{*}=\phi_{0}^{*} \quad \text { at } \quad z^{*}=0, \\
& w^{*}=0, \frac{\partial w^{*}}{\partial z^{*}}-\alpha_{2} L \frac{\partial^{2} w^{*}}{\partial z^{* 2}}=0, T^{*}=T_{u}^{*}, \phi^{*}=\phi_{u}^{*} \quad \text { at } \quad z^{*}=1 .
\end{aligned}
$$

where the parameters $\alpha_{1}$ and $\alpha_{2}$ each takes the value $\infty$ for the case of free boundary and 0 for a rigid boundary. To nondimensionalize the governing Eqs. (1)-(4), the variables are scaled as follows

$$
\begin{aligned}
& \left(x^{*}, y^{*}, z^{*}\right)=L(x, y, z), p^{*}=\frac{p \mu \kappa_{v}}{\tilde{K}_{V}}, t^{*}=\frac{t \sigma L^{2}}{\kappa_{v}}, \phi=\frac{\phi^{*}-\phi_{l}^{*}}{\phi_{u}^{*}-\phi_{l}^{*}}, \psi_{z}^{*}=\frac{\psi_{z} \kappa_{v}}{L}, t^{*}=\frac{t \sigma L^{2}}{\kappa_{v}}, T=\frac{T^{*}-T_{u}^{*}}{\Delta T^{*}}, \\
& \left(u^{*}, v^{*}, w^{*}\right)=\frac{\kappa_{v}}{L}(u, v, w),
\end{aligned}
$$

where $\kappa_{v}=\frac{\tilde{\kappa}_{m}}{(\rho c)_{f}}$ is the effective thermal diffusivity and $\sigma=\frac{(\rho c)_{m}}{(\rho c)_{f}}$ is the heat capacity ratio respectively. Substituting Eq. (9) into Eqs. (1)-(4), eliminating the pressure term from the momentum equation by operating curl twice and retaining the vertical component, we obtain

$$
\begin{aligned}
& \nabla \cdot \boldsymbol{u}_{D}=0, \\
& -\nabla p-\boldsymbol{u}_{a}-R m \hat{\boldsymbol{e}}_{z}+R d T \hat{\boldsymbol{e}}_{z}-R n_{d} \phi \hat{\boldsymbol{e}}_{z}-\sqrt{T a_{v}}\left(\boldsymbol{u}_{D} \times \hat{\boldsymbol{e}}_{z}\right)=0, \\
& \frac{\partial T}{\partial t}+\boldsymbol{u}_{D} \cdot \nabla T-\frac{\partial^{2} T}{\partial z^{2}}-\zeta \nabla_{H}^{2} T-\frac{N_{B}}{L n} \nabla \phi \cdot \nabla T-\frac{N_{A} N_{B}}{L n} \nabla T \cdot \nabla T-Q=0, \\
& \frac{1}{\sigma} \frac{\partial \phi}{\partial t}+\frac{1}{\varepsilon} \boldsymbol{u}_{D} \cdot \nabla \phi-\frac{1}{L n} \nabla^{2} \phi-\frac{N_{A}}{L n} \nabla^{2} T=0,
\end{aligned}
$$

with boundary condition

$$
\begin{array}{ll}
w=0, \frac{\partial w}{\partial z}+\alpha_{1} L \frac{\partial^{2} w}{\partial z^{2}}=0, T=1, \phi=0 & \text { at } \quad z=0, \\
w=0, \frac{\partial w}{\partial z}-\alpha_{2} L \frac{\partial^{2} w}{\partial z^{2}}=0, T=0, \phi=1 \quad \text { at } \quad z=1,
\end{array}
$$


where $\boldsymbol{u}_{a}=\left[\frac{1}{\xi} u, \frac{1}{\xi} v, w\right]$ is the anisotropic modified velocity vector, $\hat{\boldsymbol{e}}_{z}=(0,0,1)$ is the unit vector in the z-direction, $\quad R d=\frac{\rho_{f} g \alpha_{T} \tilde{K}_{V} L \Delta T^{*}}{\mu \kappa_{v}} \quad$ is the thermal Darcy-Rayleigh number, $R m=\frac{\left[\rho_{p} \phi_{l}^{*}+\rho_{f}\left(1-\phi_{l}^{*}\right)\right] g \tilde{K}_{V} L}{\mu \kappa_{v}}$ is the basic density Rayleigh number, $L n=\frac{\kappa_{v}}{D_{B}}$ is the Lewis number, $R n_{d}=\frac{\left(\rho_{p}-\rho_{f}\right)\left(\phi_{u}^{*}-\phi_{l}^{*}\right) g \tilde{K}_{V} L}{\mu \kappa_{v}}$ is the nanoparticles concentration Darcy-Rayleigh number, $T a_{v}=\left(\frac{2 \Omega^{*} \rho_{f} \tilde{K}_{V}}{\mu \varepsilon}\right)^{2}$ is the porous media related Taylor-Vadasz number, $N_{B}=\frac{\varepsilon(\rho c)_{p}}{(\rho c)_{f}}\left(\phi_{u}^{*}-\phi_{l}^{*}\right)$ is the modified particle-density increment, $N_{A}=\frac{D_{T} \Delta T^{*}}{D_{B} T_{u}^{*}\left(\phi_{u}^{*}-\phi_{l}^{*}\right)}$ is the modified density ratio, $\xi=\frac{\tilde{K}_{H}}{\tilde{K}_{V}}$ is the mechanical anisotropy parameter, $\zeta=\frac{\tilde{\kappa}_{m H}}{\tilde{\kappa}_{m V}}$ is the thermal anisotropy parameter, and $Q=\frac{Q_{0}^{*} L^{2}}{2 \kappa_{v}(\rho c)_{f} \Delta T^{*}}$ is the dimensionless heat source strength.

In the quiescent basic state, the temperature and volumetric fraction of nanoparticles vary only in the vertical z-direction and satisfy the following equations [27],

$$
\begin{aligned}
& -\frac{d p_{b}}{d z}-R m+R d T_{b}-R n_{d} \phi_{b}=0 \\
& \frac{d^{2} T_{b}}{d z^{2}}+\frac{N_{B}}{L n} \frac{d T_{b}}{d z} \frac{d \phi_{b}}{d z}+\frac{N_{A} N_{B}}{L n}\left(\frac{d^{2} T_{b}}{d z^{2}}\right)+Q=0, \\
& N_{A} \frac{d^{2} T_{b}}{d z^{2}}+\frac{d^{2} \phi_{b}}{d z^{2}}=0 .
\end{aligned}
$$

The above equations are solved subject to the boundary conditions

$$
\begin{array}{lll}
T_{b}(0)=1, \phi_{b}(0)=0 & \text { at } & z=0, \\
T_{b}(1)=0, \phi_{b}(1)=1 & \text { at } & z=1 .
\end{array}
$$

Integrating Eq. (18) with respect to $z$ and using the boundary conditions (19) and (20), we get

$$
\phi_{b}=-N_{A} T_{b}+\left(1-N_{A}\right) z+N_{A} .
$$

Using Eq. (20) in Eq. (17), we obtain 


$$
\frac{d^{2} T_{b}}{d z^{2}}+\frac{\left(1-N_{A}\right) N_{B}}{\operatorname{Ln}} \frac{d T_{b}}{d z}+Q=0
$$

On integrating (22) with respect to $z$ twice and using the boundary conditions (19) and (20), we get

$$
\begin{aligned}
& T_{b}(z)=\frac{e^{\frac{\left(N_{A}-1\right) N_{B} z}{L n}}\left[-N_{B}\left(N_{A}-1\right)-L n Q\right]-\operatorname{Ln} Q(z-1)+e^{\frac{\left(N_{A}-1\right) N_{B} z}{L n}}\left[N_{B}\left(N_{A}-1\right)-\operatorname{Ln} Q z\right]}{N_{B}\left(N_{A}-1\right)\left[e^{\frac{\left(N_{A}-1\right) N_{B} z}{L n}}-1\right]}, \\
& \phi_{b}(z)=z+\frac{N_{A}\left(N_{A} N_{B}-N_{B}+\operatorname{Ln} Q\right)\left[e^{\frac{\left(N_{A}-1\right) N_{B} z}{L n}}-1+z-z e^{\left.\frac{\left(N_{A}-1\right) N_{B} z}{L n}\right]}\right.}{N_{B}\left(N_{A}-1\right)\left[e^{\frac{\left(N_{A}-1\right) N_{B} z}{L n}}-1\right]} .
\end{aligned}
$$

For most of the nanofluids, $N_{A} \approx 1$ to $10, L n \approx 10^{2}$ to $10^{3}, N_{B} \approx 10^{-4}$ to $10^{-2}$, and consequently $\varepsilon=\frac{\left(-1+N_{A}\right) N_{B}}{L n}$ is very small of order $10^{-7}$ to $10^{-4}$ [27]. Hence, expanding $T_{b}(z)$ and $\phi_{b}(z)$ in power series of $\varepsilon$ and retaining up to the first-order terms, we have

$$
\begin{aligned}
& T_{b}(z)=\frac{1}{2}\left(2-2 z+Q z-Q z^{2}\right)+\frac{1}{12}\left(6 z-Q z-6 z^{2}+3 Q z^{2}-2 Q z^{3}\right) \varepsilon \ldots \\
& \phi_{b}(z)=\left(z-\frac{N_{A} Q z}{2}+\frac{N_{A} Q z^{2}}{2}\right)+\frac{1}{12}\left(-6 N_{A} z+N_{A} Q z+6 N_{A} z^{2}-3 N_{A} Q z^{2}+2 N_{A} Q z^{3}\right) \varepsilon+\ldots
\end{aligned}
$$

here $\varepsilon \approx 10^{-7}$ to $10^{-4}$, as compared to $\left[\frac{1}{2}\left(2-2 z+Q z-Q z^{2}\right)\right] \approx 10^{\circ}$ or $\left(z-\frac{N_{A} Q z}{2}+\frac{N_{A} Q z^{2}}{2}\right) \approx 10^{0}$, the zeroth order terms are dominant in both $T_{b}(z)$ and $\phi_{b}(z)$, hence approximately, we proceed with

$$
\begin{aligned}
& T_{b}(z)=\frac{1}{2}\left(2-2 z+Q z-Q z^{2}\right), \\
& \phi_{b}(z)=\left(z-\frac{N_{A} Q z}{2}+\frac{N_{A} Q z^{2}}{2}\right),
\end{aligned}
$$

which demonstrate quadratic distributions in $z$ [27].

Suppose that the basic state is disturbed by an infinitesimal thermal perturbation. We now superimpose perturbations on the basic solution. We write 


$$
(u, v, w, p, T, \phi, \psi)=\left[0,0,0, p_{b}(z), T_{b}(z), \phi_{b}(z), \psi_{b, z}\right]+\left(u^{\prime}, v^{\prime}, w^{\prime}, p^{\prime}, T^{\prime}, \phi^{\prime}, \psi_{z}^{\prime}\right) .
$$

We substitute Eq. (29) into Eqs. (10)-(13) and linearize them by neglecting the products of primed quantities and obtain

$\nabla \cdot \boldsymbol{u}_{D}^{\prime}=0$

$-\nabla p^{\prime}-\boldsymbol{u}_{a}^{\prime}+R d T^{\prime} \hat{\boldsymbol{e}}_{z}-R n_{d} \phi^{\prime} \hat{\boldsymbol{e}}_{z}-\sqrt{T a_{v}}\left(\boldsymbol{u}_{D}^{\prime} \times \hat{\boldsymbol{e}}_{z}\right)=0$,

$\frac{\partial T^{\prime}}{\partial t}-w^{\prime} \frac{\partial T_{b}}{\partial t}=\frac{\partial^{2} T^{\prime}}{\partial z^{2}}+\zeta \nabla_{H}^{2} T^{\prime}-2 \frac{N_{A} N_{B}}{L n} \frac{\partial T^{\prime}}{\partial z} \frac{\partial T_{b}}{\partial z}+\frac{N_{B}}{L n} \frac{\partial T^{\prime}}{\partial z} \frac{\partial \phi_{b}}{\partial z}-\frac{N_{B}}{L n} \frac{\partial \phi^{\prime}}{\partial z} \frac{\partial T_{b}}{\partial z}=0$,

$\frac{1}{\sigma} \frac{\partial \phi^{\prime}}{\partial t}+\frac{1}{\varepsilon} \frac{\partial \phi_{b}}{\partial z} w^{\prime}=\frac{1}{L n} \nabla^{2} \phi^{\prime}+\frac{N_{A}}{L n} \nabla^{2} T^{\prime}$

Taking curl $\hat{\boldsymbol{e}}_{z} \cdot \operatorname{curl}(\nabla \times)$ of Eq. (31), and retaining the z-component, we obtain

$\frac{1}{\xi} \psi_{z}^{\prime}+\sqrt{T a_{v}} \frac{\partial w^{\prime}}{\partial z}=0$

Now, taking the curl $(\nabla \times)$ twice on the resulting of Eq. (31) together with Eq. (30) by using the curl identity, retaining the vertical component and can be simplified with Eq. (34) as below

$\left[\nabla_{H}^{2}+\frac{1}{\xi} \frac{\partial^{2}}{\partial z^{2}}-\xi T a_{v} \frac{\partial^{2}}{\partial z^{2}}\right] w^{\prime}-R d \nabla_{H}^{2} T^{\prime}+R n_{d} \nabla_{H}^{2} \phi^{\prime}=0$,

where $\psi_{z}^{\prime}$ is simplified and vanished.

The normal mode expansion of the dependent variables is assumed in the form

$$
\left.\left(w^{\prime}, T^{\prime}, \phi^{\prime}\right)=[W(z), \Theta(z), \Phi(z)] e^{\left[i\left(a_{x} x+a_{y} y\right)+s t\right.}\right],
$$

then, substituting Eq. (36) into Eqs. (32)-(35) and neglecting the terms of the second and higher orders in the perturbations, we obtain

$$
\begin{aligned}
& \left(\frac{1}{\xi} D^{2}-a^{2}+\xi T a_{v} D^{2}\right) W+a^{2} R a \Theta-a^{2} R n_{d} \Phi=0, \\
& \frac{1}{2}(-2+Q-2 Q z) W+\left[D^{2}-\zeta a^{2}+\frac{N_{A} N_{B}}{L n}(-2+Q-2 Q z) D+\frac{N_{B}}{L n}\left(1-\frac{1}{2} N_{A} Q+N_{A} Q z\right) D\right] \Theta- \\
& \frac{1}{2} \frac{N_{B}}{L n}(-2+Q-2 Q z) D \Phi=0,
\end{aligned}
$$




$$
-\frac{1}{\varepsilon}\left(1-\frac{1}{2} N_{A} Q+N_{A} Q z\right) W-\frac{N_{A}}{L n}\left(D^{2}-a^{2}\right) \Theta-\frac{1}{L n}\left(D^{2}-a^{2}\right) \Phi=0,
$$

where $a=\sqrt{a_{x}^{2}+a_{y}^{2}}$ is the wavenumber and $D=\frac{d}{d z}$. For neutral stability the real part of $s$ is zero. Hence, $s=i \omega$ where $\omega$ is real and is a dimensionless frequency.

Following the proportional feedback control as reported by Bau [36], the continuously distributed actuators and sensors are arranged in a way that for every sensor, there is an actuator positioned directly beneath it. The determination of a control, $q(t)$ can be accomplished using the proportionalintegral-differential (PID) controller of the form

$$
q(t)=r+K[e(t)], e(t)=\hat{m}(t)+m(t)
$$

where $r$ is the calibration of the control, $e(t)=\hat{m}(t)+m(t)$ is an error or deviation from the measurement, $\hat{m}(t)$, from some desired reference value, $\hat{m}(t)$, while $K$ is the scalar controller gain where $K=K_{P}+K_{D} \frac{d}{d t}+K_{L} \int_{0}^{t} d t$, and $K_{P}$ is the proportional gain, $K_{D}$ is the differential gain, and $K_{L}$ is the integral gain. Based on Eq. (40), for one sensor plane and proportional feedback control, the actuator modifies the heated surface temperature using a proportional relation between the upper, $z=1$ and the lower, $z=0$, thermal boundaries for the perturbation field

$$
T^{\prime}(x, y, 0, t)=-K T^{\prime}(x, y, 1, t)
$$

where $T^{\prime}$ denotes the deviation of the temperature of fluid from its conductive state.

Eqs. (37)-(39) are solved subject to the appropriate boundary conditions. Considering the proportional controller, $K$ positioned at the lower boundary of nanofluid layer, we will have

$$
W=D W=\Theta(0)+K \Theta(1)=\phi=\Psi=D \Psi=0 \text { at } z=0
$$

The Galerkin-type weighted residuals method is applied to find the approximate solution to the system. The variables are written in a series of basis functions

$$
W=\sum_{i=1}^{n} A_{i} W_{i}, \Theta=\sum_{i=1}^{n} B_{i} \Theta_{i}, \Phi=\sum_{i=1}^{n} C_{i} \Phi_{i}
$$

where $A_{i}, B_{i}$ and $C_{i}$ are constants and the basis functions $W_{i}, \Theta_{i}$ and $\Phi_{i}$ where $i=1,2,3, \ldots$, will be chosen corresponding to the free-free, rigid-free and rigid-rigid lower-upper boundary conditions as discussed by Khalid et al., [42].

$$
\begin{aligned}
& W_{i}=z-2 z^{3}+z^{4}, \Theta_{i}=z(z-2), \Phi_{i}=z(z-1), \\
& W_{i}=z^{2}(1-z)(3-2 z), \Theta_{i}=z(z-2), \Phi_{i}=z(z-1), \\
& W_{i}=z^{2}(1-z)^{2}, \Theta_{i}=z(z-2), \Phi_{i}=z(z-1) .
\end{aligned}
$$


Substitute Eq. (44) into Eqs. (37)-(39) and make the expressions on the left-hand sides of those equations (the residuals) orthogonal to the trial functions, thereby obtaining a system of $3 \mathrm{~N}$ linear algebraic equations in the $3 N$ unknowns. The vanishing of the determinant of the coefficients produces the eigenvalue equation for the system. One can regard $R d$ as the eigenvalue and thus $R d$ is found in terms of the other parameters.

Maple programming is used to approximate the solutions and the eigenvalue of thermal DarcyRayleigh number, $R d$ for lower-upper rigid-rigid boundary conditions has to be extracted from the following determinant definition

$$
\operatorname{det}=\left|\begin{array}{ccc}
\frac{a^{2}}{630}+\frac{2}{105 \xi}+\frac{2 T a_{v} \xi}{105} & \frac{a^{2} R d}{42} & \frac{-a^{2} R n_{d}}{140} \\
\frac{Q}{840}+\frac{1}{42} & \frac{8 a^{2} \zeta}{15}-\frac{7 Q N_{A} N_{B}}{30 L n}+\Lambda_{1} & \frac{-11 Q N_{B}}{60 L n}+\Lambda_{2} \\
\frac{Q N_{A}}{280 \varepsilon}+\Lambda_{3} & \frac{11 a^{2} N_{A}}{20 L n}+\Lambda_{4} & \frac{3 a^{2}}{10 L n}+\Lambda_{5}
\end{array}\right|
$$

here, we have

$$
\begin{aligned}
& \Lambda_{1}=\frac{(Q-2) N_{A} N_{B}}{2 L n}+\frac{2-Q N_{A}}{2 L n} N_{B}+\frac{4}{3}+K, \\
& \Lambda_{2}=\frac{-2+Q}{6 L n} N_{B}, \\
& \Lambda_{3}=\frac{2-Q N_{A}}{140 \varepsilon}, \\
& \Lambda_{4}=\frac{2 N_{A}-2 a^{a} N_{A}}{3 \operatorname{Ln}}-\frac{N_{A}}{L n}, \\
& \Lambda_{5}=\frac{2-a^{2}}{3 L n}-\frac{1}{\operatorname{Ln}} .
\end{aligned}
$$

\section{Results and Discussion}

Control on the onset of Rayleigh-Benard convection is investigated in a rotating anisotropic porous medium (the Horton-Rogers-Lapwood problem) saturated by nanofluids with internal heat source heated from below. Three different types of velocity boundary conditions, namely both boundaries free (free-free), both boundaries rigid (rigid-rigid) and lower rigid-upper free (rigid-free) are considered. The resulting generalized eigenvalue problem is solved numerically using the Galerkin method. The parameters are fixed with $K=10, T a_{v}=500, \varepsilon=0.9, Q=0.5, \xi=0.8, \zeta=0.6, N_{A}=2$, $N_{B}=0.01, L n=100$ and $R n_{d}=2$ except the varying parameter. Figures 2-9 are the plotted for the configuration of Darcy-Rayleigh number $R d$ versus wavenumber $a$, and in these respective figures, the comparative analysis of the thermal instability for porosity $\varepsilon=0.1$ and 0.9 are done. Meanwhile, Figures 10-15 show the plots for critical Darcy-Rayleigh number, $R d_{c}$ as a function of selected parameters.

Test computations have been performed and the results are compared with Char and Chiang [24] and Yadav et al., [27] for the limiting case of nanofluids (regular fluids, $R a_{c}$ ) $K=0, T a_{v}=0, Q=0, \xi=$ 
$0, \zeta=0, N_{A}=, N_{B}=0.01, L n=0, R n_{d}=0$ in the absence of porous media, $\varepsilon=0$. The comparison results of critical thermal Rayleigh number $R a_{c}$ for the rigid-rigid, rigid-free and free-free boundary conditions are presented in Tables 1-3. As can be seen in the tables, the results are in good agreement with those reported by Char and Chiang [24] and Yadav et al., [27], thus verify the accuracy of the analysis.

Table 1

Comparisons of $R a_{c}$ for different values of $Q$ for regular fluid in rigid-rigid boundary conditions

\begin{tabular}{llll}
\hline \multirow{2}{*}{$Q$} & Char and Chiang [24] & Yadav et al., [27] & Present Study \\
\cline { 2 - 4 } & Rigid-rigid & Rigid-rigid & Rigid-rigid \\
& $R a_{c}$ & $R a_{c}$ & $R a_{c}$ \\
\hline 0 & 1707.85 & 1707.76 & 1707.85 \\
1 & 1704.61 & 1704.52 & 1705.41 \\
2 & 1695.04 & 1694.95 & 1702.97 \\
10 & 1463.05 & 1462.86 & 1463.13 \\
20 & 1118.66 & 1118.45 & 1118.35 \\
\hline
\end{tabular}

\section{Table 2}

Comparisons of $R a_{c}$ for different values of $Q$ for regular fluid in rigid-free boundary conditions

\begin{tabular}{llll}
\hline & Char and Chiang [24] & Yadav et al., [27] & Present Study \\
\cline { 2 - 4 }$Q$ & Rigid-free & Rigid-free & Rigid-free \\
& $R a_{c}$ & $R a_{c}$ & $R a_{c}$ \\
\hline 0 & 1100.65 & 1100.64 & 1100.65 \\
1 & 1055.58 & 1055.57 & 1057.46 \\
2 & 1011.44 & 1011.43 & 1017.53 \\
10 & 725.60 & 725.60 & 725.59 \\
20 & 517.87 & 517.83 & 517.73 \\
\hline
\end{tabular}

Table 3

Comparisons of $R a_{c}$ for different values of $Q$ for regular fluid in free-free boundary conditions

\begin{tabular}{lll}
\hline \multirow{2}{*}{$Q$} & Yadav et al., [27] & Present Study \\
\cline { 2 - 3 } & Free-free & Free-free \\
& $R a_{c}$ & $R a_{c}$ \\
\hline 0 & 1707.76 & 1707.85 \\
1 & 1704.52 & 1705.41 \\
2 & 1694.95 & 1702.97 \\
10 & 1462.86 & 1463.13 \\
20 & 1118.45 & 1118.35 \\
\hline
\end{tabular}

In Figure 2 showed the variation of thermal Darcy-Rayleigh number, $R d$ with wavenumber, $a$ for different values of the mechanical anisotropy parameter, $\xi=0.7$ and 0.8 . It is found from Figure 2 that as $\xi$ increases the value of thermal Darcy-Rayleigh number, $R d$ also increases showing a stabilizing effect on thermal instability. This is due to the permeability $\tilde{K}_{H}$ in the $x$-direction increases or permeability in the $z$-direction $\tilde{K}_{V}$ decreases. Therefore, in both situations it becomes more difficult for the fluid to flow in the $z$-direction and, hence, onset of convection takes place at a higher thermal Darcy-Rayleigh number, $R d$. As for the Figure 3 indicates the effect of thermal anisotropy parameter, $\zeta=0.6,0.7$ on the instability. The thermal Darcy-Rayleigh number, $R d$ increases on 
increasing thermal anisotropy parameter, $\zeta$ showing that the effect of increasing the thermal anisotropy parameter, $\zeta$ is to stabilize the system. As for the effect of porosity, it is found to stabilize the system. From the respective figure, the increase in porosity can be defined as larger volume of the void spaces, hence the flows of nanofluids are slowed down causing the onset of instability to be delayed [27]. Therefore, it is observed that by increasing the porosity is to increase Darcy-Rayleigh number, $R d$ and to stabilize the system as reported by Yadav et al., [27].

The plot of thermal Darcy-Rayleigh number, $R d$ and the corresponding wavenumber, $a$ for the selected values of feedback control, $K=15$ and 20 is presented in Figure 4 for two values of porosity, $\varepsilon=0.1$ and 0.9 respectively. Physically, the sensors detect the departure of the fluid from its conductive state and then they direct the actuators to take action so as to suppress any disturbances [36]. In this figure, the results obtained for three different types of velocity boundary conditions, rigid-rigid, rigid-free, and free-free are compared. It is noted that, the rigid-rigid boundaries lead to a substantial stabilizing effect compared to rigid-free and free-free boundaries.

Figure 5 shows the variation of Taylor-Vadasz number, $T a_{v}=700$ and 750 for two values of porosity, $\varepsilon=0.1$ and 0.9 in the plot of thermal Darcy-Rayleigh number, $R d$ versus wavenumber, $a$ respectively. The thermal Darcy-Rayleigh number, $R d$ increases with the increased in Taylor-Vadasz number, $T a_{v}$, indicating that the Coriolis force due to a rotation inhibits the onset of convection in nanofluids layer. The fluid moves to the horizontal plane with higher velocity because of the vorticity introduced by the rotation mechanism. Therefore, the velocity of the fluids in the vertical plane is reduced, thereby minimizing the amount of thermal convection [40].

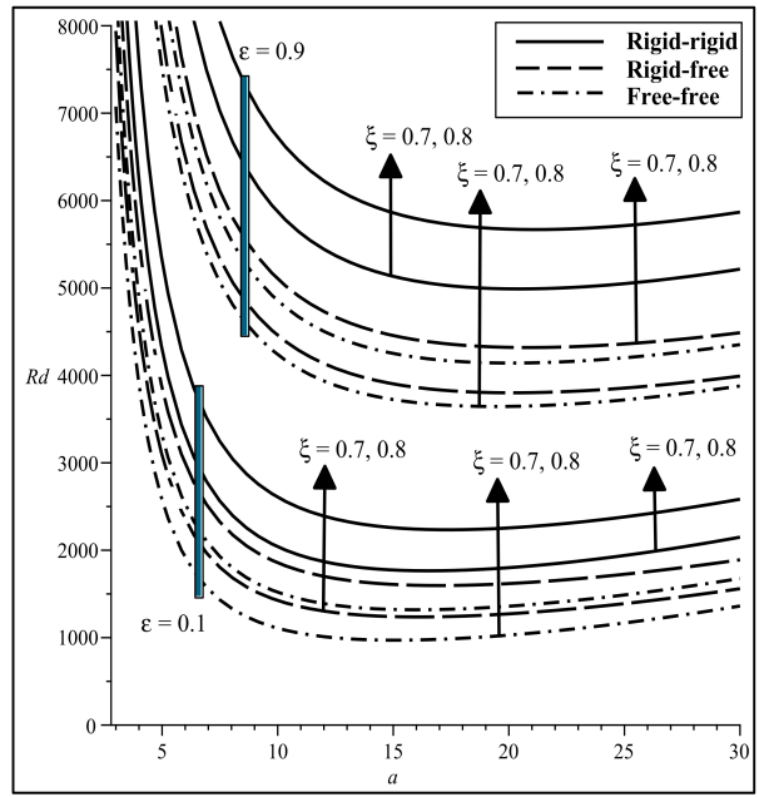

Fig. 2. Plot of $\xi$ on $R d$ against $a$

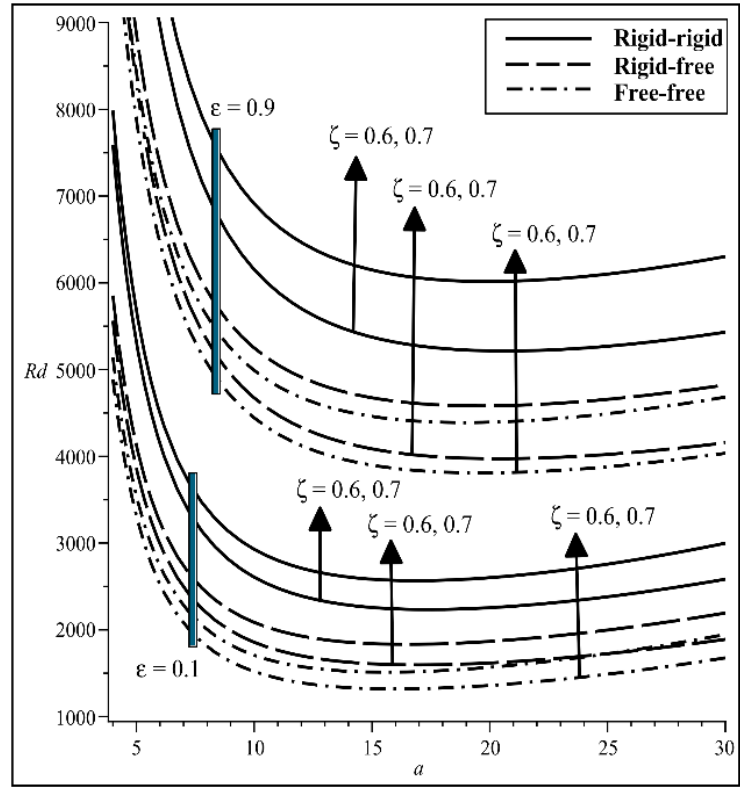

Fig. 3. Plot of $\zeta$ on $R d$ against $a$ 


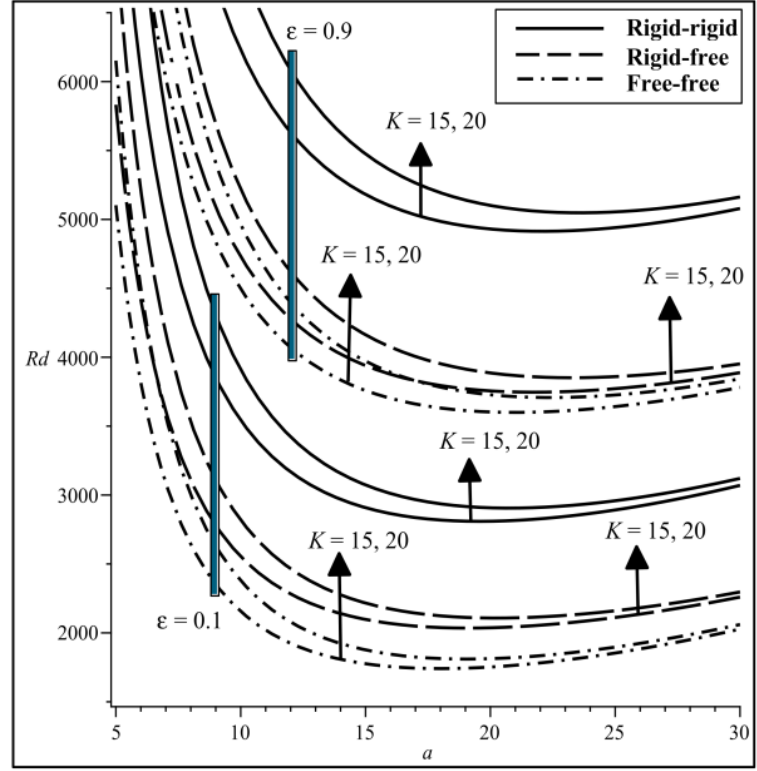

Fig. 4. Plot of $K$ on Rd against a

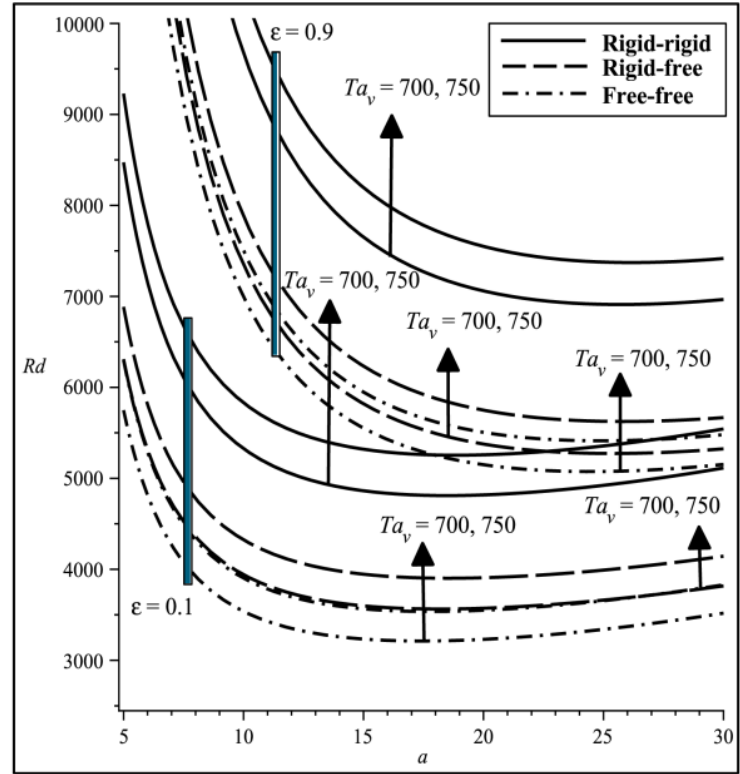

Fig. 5. Plot of Tav on Rd against a

The presence of internal heat source, $Q$ have a significant influence on the thermal instability of the system. To see the effect of internal heat source strength, $Q$ on the criterion for the onset of thermal convection in nanofluids, Figure 6 shows the plot for different values of internal heat source, $Q=0.5,1$ when porosity, $\varepsilon=0.1,0.9$ in three types of boundary conditions. This plot shows the behaviour of internal heat source, $Q$ which the thermal Darcy-Rayleigh number, $R d$ value is decreasing as internal heat source strength, $Q$ is increased. The increase in the internal heat source strength, $Q$ amount is to increase in energy supply to the system. This gives large deviations in the distributions which in turn improve the disturbances in the layer and the system becomes more unstable $[24,27,28]$.

Figure 7 shows the Darcy-Rayleigh number, $R d$ versus the wavenumber, $a$ for various values of the nanoparticles concentration Darcy-Rayleigh number, $R n_{d}=1$ and 2 when porosity $\varepsilon=0.1$ and 0.9 in various boundary conditions. Theoretically, an increase of nanoparticles concentration DarcyRayleigh number, $R n_{d}$ increase the density of the nanoparticles, according to the definition nanoparticles concentration Darcy-Rayleigh number, $R n_{d}$ and enhance the heat transfer diffusion within the nanofluid layer. In Figure 8, the chosen values for nanofluids Lewis number, $L n=100$ and 150 are plotted for $\varepsilon=0.1$ and 0.9 respectively. The effect of increasing nanofluids Lewis number, $L n$ advances the onset of convective instability as thermal diffusion is dominated over Brownian diffusion according to the definition of nanofluids Lewis number, $L n$.

Thermal Darcy-Rayleigh number, $R d$ against wavenumber, $a$ is plotted in Figure 9 for modified diffusivity ration, $N_{A}=1$ and 9 when $\varepsilon=0.1$ and 0.9 in three types of boundary conditions. It is found that, thermal Darcy-Rayleigh number, $R d$ decreases with an increase of modified diffusivity ratio, $N_{A}$ due to the directly proportional relationship between parameter $N_{A}$ to the thermophoretic diffusion coefficient $D_{T}$. Although thermophoresis effect and Brownian motion are responsible for the motion of nanoparticles in the base fluids, it is observed that thermophoretic effect is more dominating in order to initiate the diffusion of nanoparticles. Therefore, the increase in the values of $N_{A}$ increase the temperature difference within the nanofluids layer thus hasten the onset of convection. It is interesting to note that the modified particle density, $N_{B}$ has no significant effect on the nanofluids system. An attempt has been made to scrutinize the effect of $N_{B}$ in this study but there is no apparent result. This finding agrees well with those reported by Yadav et al., [27] that the impact of $N_{B}$ is so small and can be omitted. 


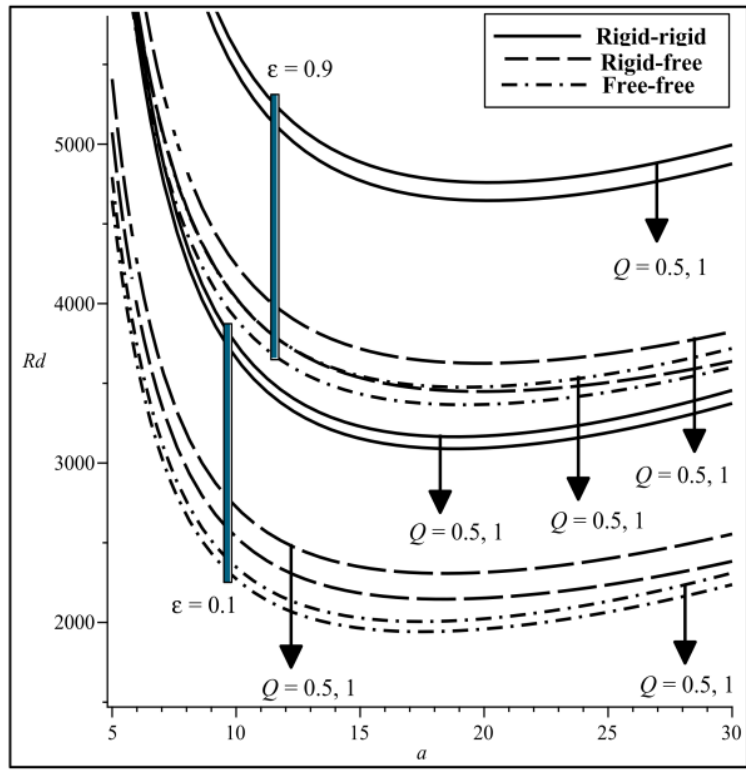

Fig. 6. Plot of $Q$ on $R d$ against $a$

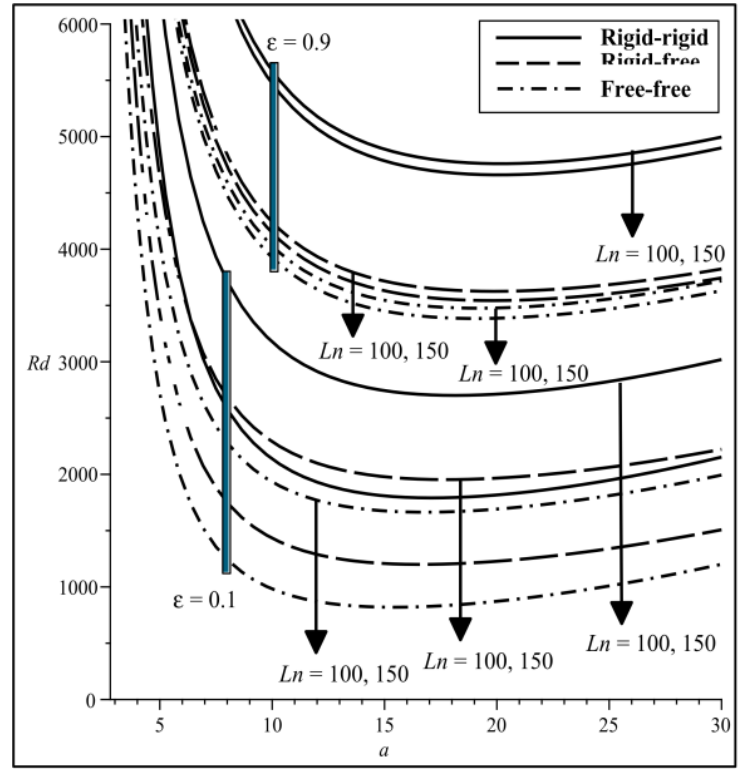

Fig. 8. Plot of $L n$ on $R d$ against $a$

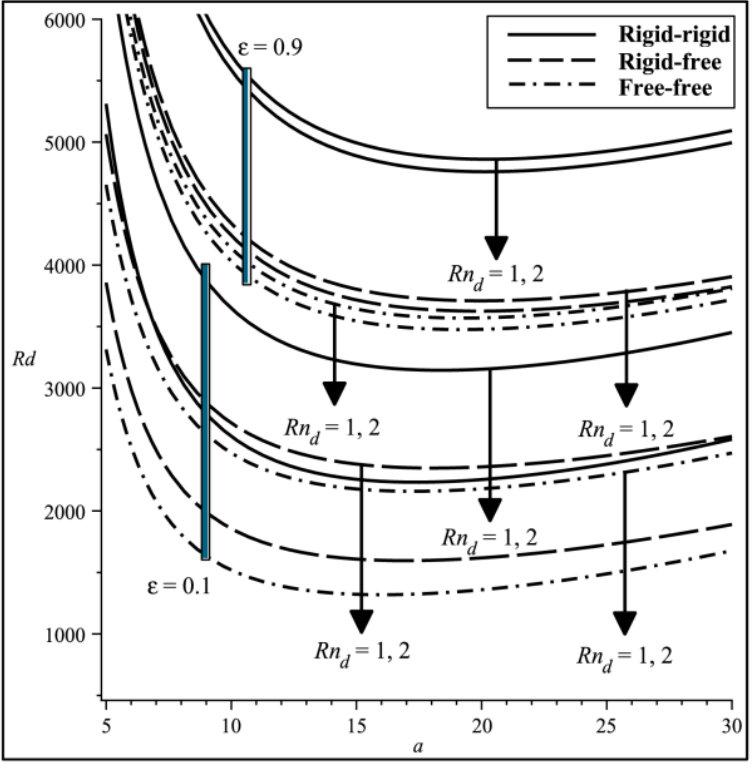

Fig. 7. Plot of $R n_{d}$ on $R d$ against $a$

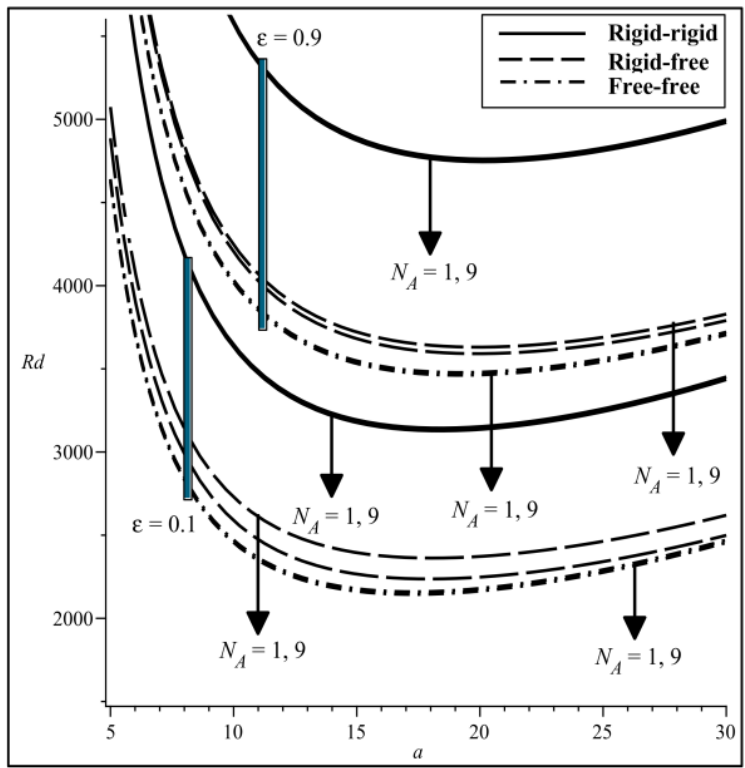

Fig. 9. Plot of $N_{A}$ on $R d$ against $a$

The influences of the Taylor-Vadasz number, $T a_{v}$ to the nanoparticles concentration DarcyRayleigh number, $R n_{d}=1,9$ are presented in Figure 10 respectively. Obviously, increasing the Coriolis force due to the rotation in nanofluids layer helps to reduce the rate of disturbance caused by nanoparticles concentration Darcy-Rayleigh number, $R n_{d}$ thus promoting the stability within the nanofluids layer system. Scrutinizing the critical thermal Darcy-Rayleigh number, $R d_{c}$ with different boundary conditions, the rigid-rigid boundaries maintain gaining the highest values of $R d_{c}$ compared to the free-free and rigid-free boundaries.

The graphs of critical thermal Darcy-Rayleigh number, $R d_{c}$ against feedback control, $K$ for selected values of internal heat source, $Q=0.5,1.5$ are depicted in Figure 11, respectively. As illustrated earlier, the impact of increasing internal heat source, $Q$ advanced the process of thermal instability. However, elevating the values of feedback control, $K$ helps to delay the onset of convection induced by the effect of internal heat source and stabilizes the system. Feedback control suppress the rate of thermal instability due to the destabilize effect of internal heat source within the system. 
The variation of critical thermal Darcy-Rayleigh number, $R d_{c}$ as a function of nanofluids Lewis number, $L n$ is presented in Figure 12 for the values of Taylor-Vadasz number, $T a_{v}=1000,2000$ respectively. In the figure, the results obtained for three different types of velocity boundary conditions, namely rigid-rigid, rigid-free and free-free are compared. It is found that, the stabilizing effect of Taylor-Vadasz number, $T a_{v}$ is significantly altered by the destabilizing effect of $L n$. Increasing the values of $L n$ leads to the high diffusivity rate of heat transfer mechanism thus promoting a destabilization phenomenon within the system.

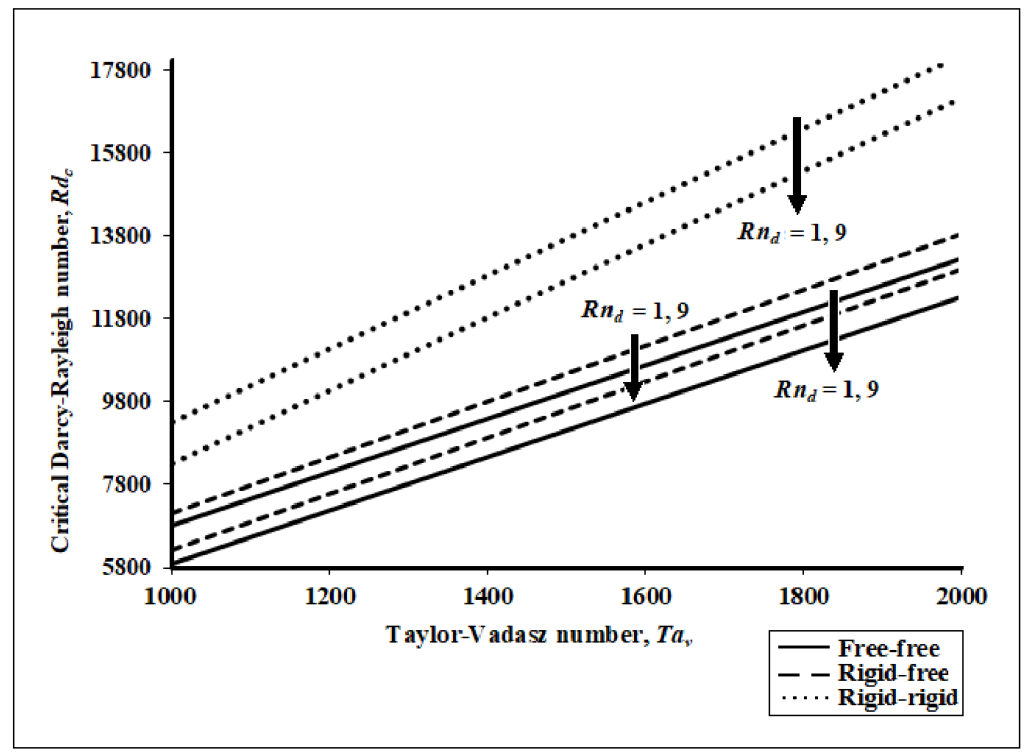

Fig. 10. Plot of $R d_{c}$ with $T a_{v}$ for selected values of $R n_{d}$

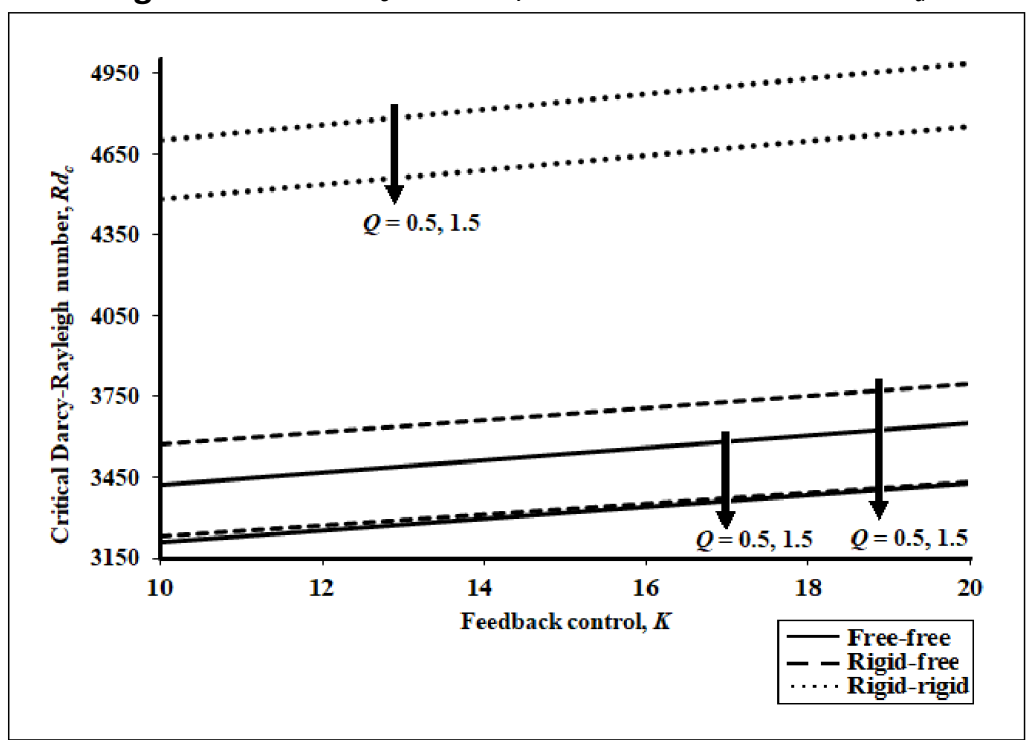

Fig. 11. Plot of $R d_{c}$ with $K$ for selected values of $Q$ 


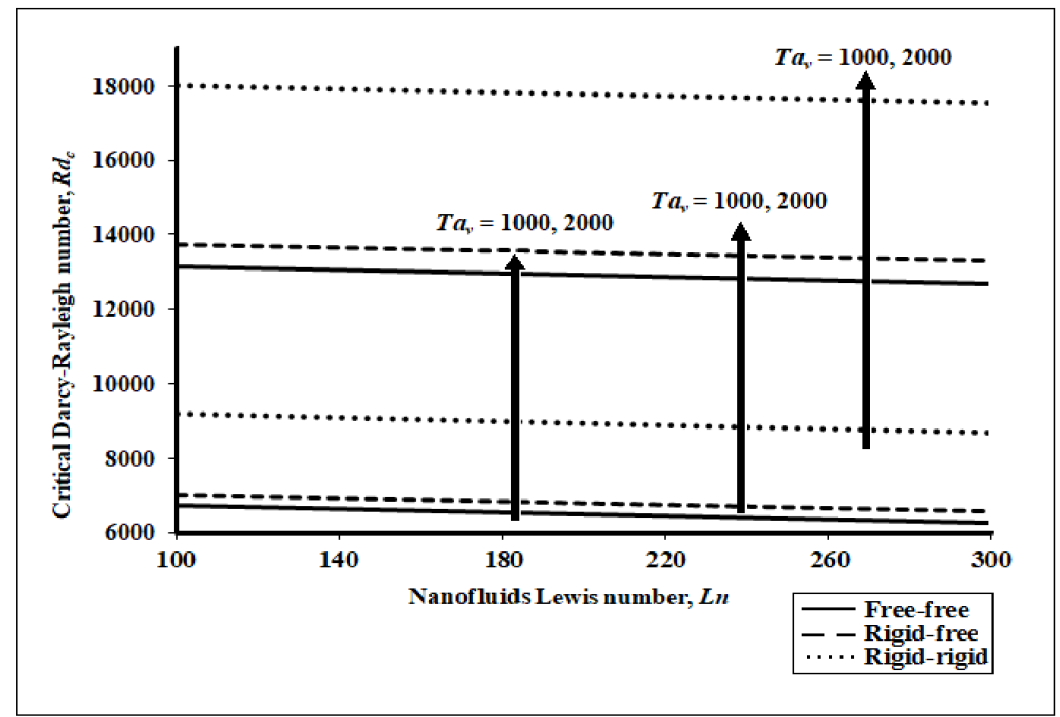

Fig. 12. Plot of Rdc with $\mathrm{Ln}$ for selected values of Tav

The impact of anisotropic parameters on the onset of convection is depicted in Figure 13. The variation of critical thermal Darcy-Rayleigh number, $R d_{c}$ against the thermal anisotropy parameter, $\zeta$ and mechanical anisotropy parameter, $\xi$ is shown in Figure 13. The effect of increasing thermal anisotropy parameter, $\zeta$ and mechanical anisotropy parameter, $\xi$ is to respectively slow down the onset of convection thus stabilized the system. It is found that the effect of the mechanical anisotropy parameter, $\xi$ is more dominant than thermal anisotropy parameter, $\zeta$. Therefore, the permeability in the $z$-direction $\tilde{K}_{V}$ decreases, thus the fluid flow in the $z$-direction is reduced.

Finally, from Figures 14 and 15, it is observed that the onset of convection is depends on the values of porosity subjected to the porous medium, where increasing the values of porosity, $\varepsilon$ delays the convection for the increasing values of feedback control, $K=10,30$ and internal heat source, $Q=$ $0.5,1.5$. The results show that, the porosity significantly stabilized the system in the presence of stabilizing effect of feedback control, $K$ and destabilizing effect of internal heat source, $Q$. Increasing the values of porosity lead to the increase in the volume of void spaces, therefore, the flows of nanofluids are slowed down causing the onset of instability to be postponed [27] even in the existence of internal heat source.

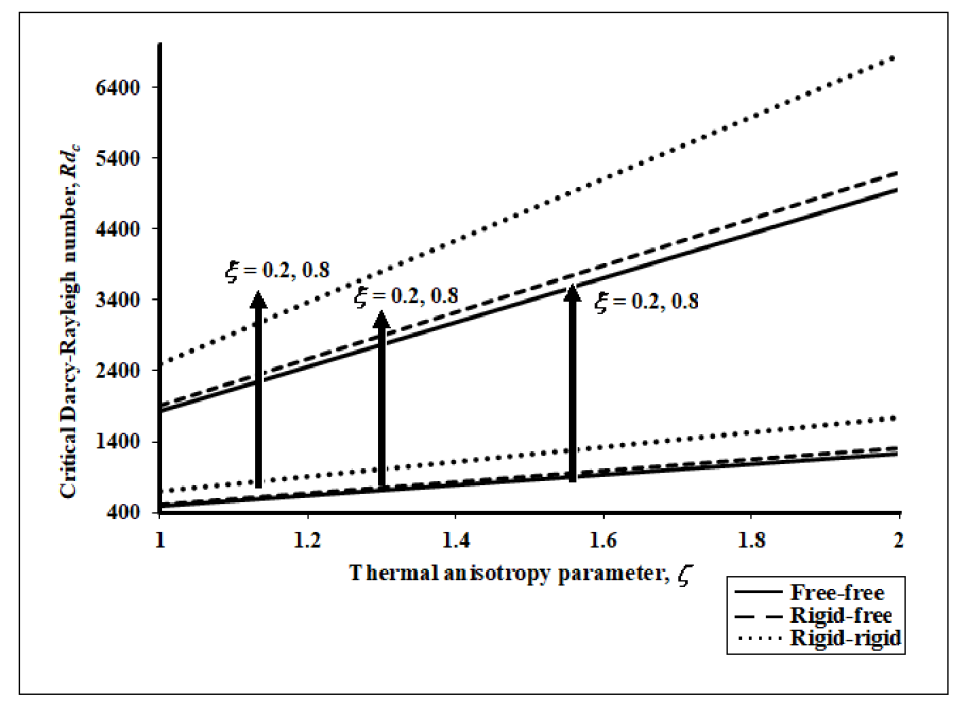

Fig. 13. Plot of Rdc with $\zeta$ for selected values of $\xi$ 


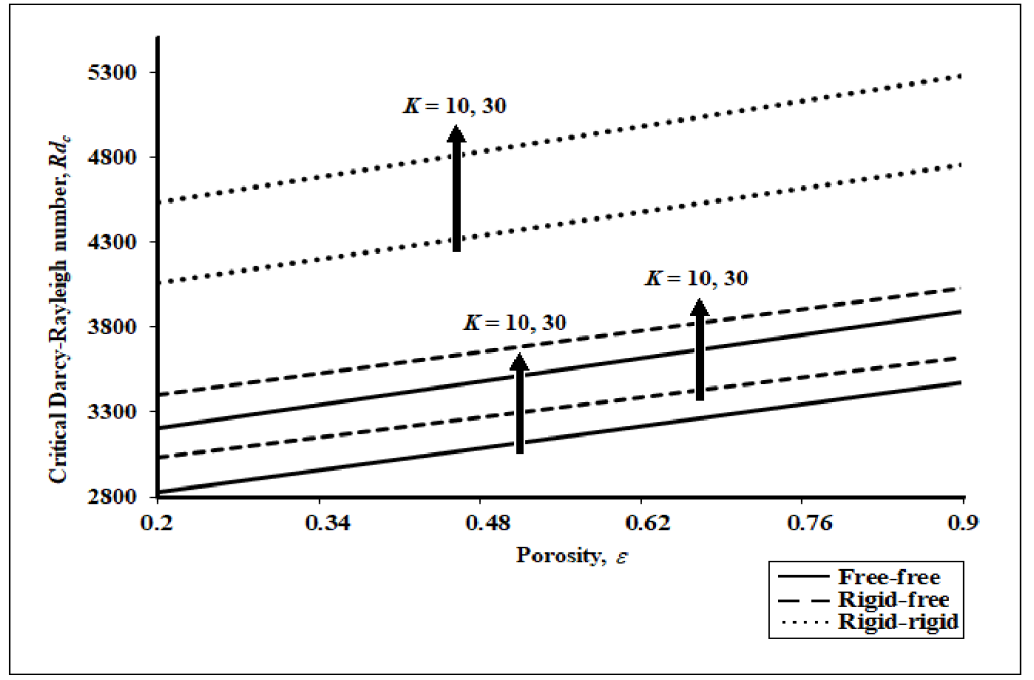

Fig. 14. Plot of Rdc with $\varepsilon$ for selected values of K

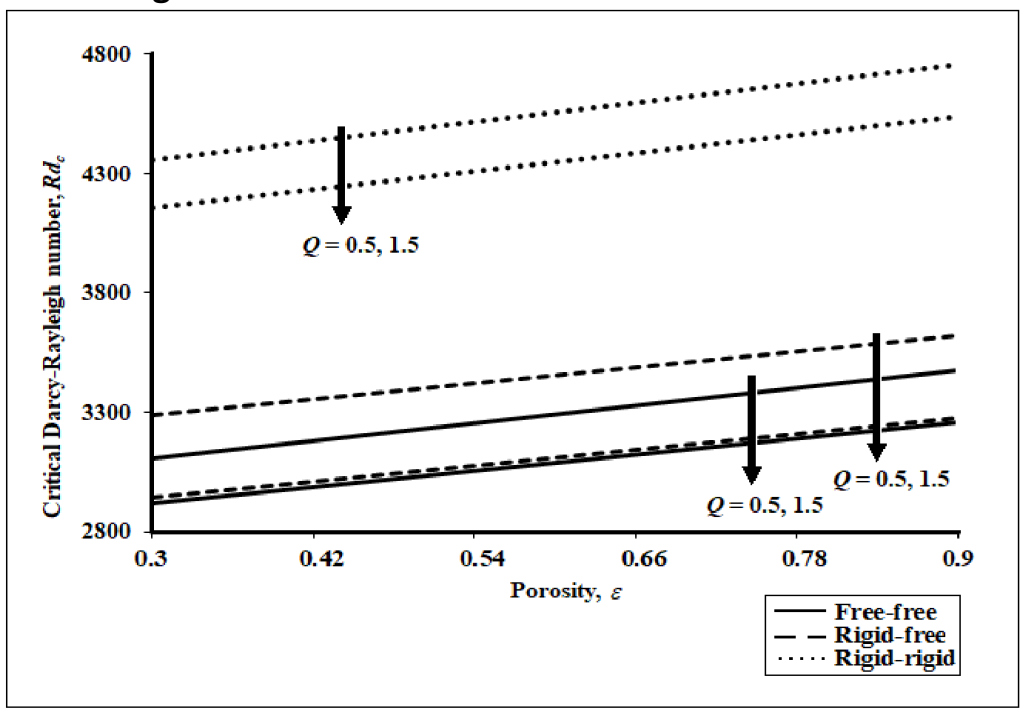

Fig. 15. Plot of $R d_{c}$ with $\varepsilon$ for selected values of $Q$

\section{Conclusions}

Linear stability analysis of Rayleigh-Benard convective instability in nanofluids layer saturating a rotating anisotropic porous medium (the Horton-Rogers-Lapwood problem) heated from below with feedback control and internal heat source has been examined theoretically. The Darcy model includes the effective mechanism for convective enhancement in nanofluids layer system, namely the Brownian motion and thermophoresis. Free-free, rigid-rigid, and lower-rigid and upper-free boundary conditions are taken in consideration. The resulting eigenvalue problem is solved numerically using the Galerkin technique and computed by using Maple software. The effect of various parameters is considered and presented graphically.

The initiation of Rayleigh-Benard convection sets in at higher values of Darcy-Rayleigh number $R d$ for the application effects of feedback control $K$, Taylor-Vadasz number $T a_{v}$, and porosity $\varepsilon$, together with the impact of anisotropic parameters; the mechanical anisotropy parameter $\xi$ and thermal anisotropy parameter $\zeta$ within the nanofluids layer system successfully. The initiation of RayleighBenard convection sets earlier when the values of internal heat source $Q$, modified diffusivity ratio 
$N_{A}$, nanofluids Lewis number $L n$ and nanoparticles concentration Darcy-Rayleigh number $R n_{d}$ are increased in the system, thus advancing the onset of Rayleigh-Benard convection.

The modified particle density increment $N_{B}$ is found to have no visible observation on the onset of convective instability in a porous medium, therefore this effect can be omitted as reported by Yadav et al., [27]. The most stable system is when the both lower-upper boundary conditions are rigid and least stable is when both lower-upper boundary conditions are free: $(R d)_{\text {free-free }}<(R d)_{\text {rigid-free }}$ $<(R d)_{\text {rigid-rigid and }}\left(R d_{c}\right)_{\text {free-free }}<\left(R d_{c}\right)_{\text {rigid-free }}<\left(R d_{c}\right)_{\text {rigid-rigid. }}$

\section{Acknowledgement}

The authors would like to express their gratitude to the Faculty of Computer and Mathematical Sciences, Universiti Teknologi MARA, Shah Alam Selangor, Malaysia.

\section{References}

[1] Choi, S. US, and Jeffrey A. Eastman. Enhancing thermal conductivity of fluids with nanoparticles. No. ANL/MSD/CP84938; CONF-951135-29. Argonne National Lab., IL (United States), 1995.

[2] Menni, Younes, Ali J. Chamkha, Giulio Lorenzini, Noureddine Kaid, Houari Ameur, and Mohammed Bensafi. "Advances of nanofluids in solar collectors-a review of numerical studies." Math Model Eng Probl 6, no. 3 (2019): 415-27. https://doi.org/10.18280/mmep.060313

[3] Barber, Jacqueline, David Brutin, and Lounes Tadrist. "A review on boiling heat transfer enhancement with nanofluids." Nanoscale research letters 6, no. 1 (2011): 1-16. https://doi.org/10.1186/1556-276X-6-280

[4] Yu, Wei, and Huaqing Xie. "A review on nanofluids: preparation, stability mechanisms, and applications." Journal of nanomaterials 2012 (2012). https://doi.org/10.1155/2012/435873

[5] Goharshadi, E. K., Hossein Ahmadzadeh, Sara Samiee, and Mahboobeh Hadadian. "Nanofluids for heat transfer enhancement-a review." (2013): 1-33. https://doi.org/10.22036/pcr.2013.27911

[6] Mahbubul, I. M., Rahman Saidur, and M. A. Amalina. "Latest developments on the viscosity of nanofluids." International Journal of Heat and Mass Transfer 55, no. 4 (2012): 874-885. https://doi.org/10.1016/j.ijheatmasstransfer.2011.10.021

[7] Hussein, Adnan M., K. V. Sharma, R. A. Bakar, and K. Kadirgama. "A review of forced convection heat transfer enhancement and hydrodynamic characteristics of a nanofluid." Renewable and Sustainable Energy Reviews 29 (2014): 734-743. https://doi.org/10.1016/i.rser.2013.08.014

[8] Tzou, Da Yu. "Instability of nanofluids in natural convection." Journal of Heat Transfer 130, no. 7 (2008). https://doi.org/10.1115/1.2908427

[9] Tzou, Da Yu. "Thermal instability of nanofluids in natural convection." International Journal of Heat and Mass Transfer 51, no. 11-12 (2008): 2967-2979. https://doi.org/10.1016/j.ijheatmasstransfer.2007.09.014

[10] Buongiorno, Jacopo. "Convective transport in nanofluids." (2006): 240-250. https://doi.org/10.1115/1.2150834

[11] Kim, Jake, Yong Tae Kang, and Chang Kyun Choi. "Analysis of convective instability and heat transfer characteristics of nanofluids." Physics of fluids 16, no. 7 (2004): 2395-2401. https://doi.org/10.1063/1.1739247

[12] Nield, D. A., and Andrey V. Kuznetsov. "The onset of convection in a horizontal nanofluid layer of finite depth." European Journal of Mechanics-B/Fluids 29, no. $3 \quad$ (2010): 217-223. https://doi.org/10.1016/i.euromechflu.2010.02.003

[13] Yadav, Dhananjaya, G. S. Agrawal, and Rama Bhargava. "Rayleigh-Benard convection in nanofluids." International Journal of Applied Mathematics and Mechanics 7, no. 2 (2011): 61-76.

[14] Haddad, Zoubida, Eiyad Abu-Nada, Hakan F. Oztop, and Amina Mataoui. "Natural convection in nanofluids: are the thermophoresis and Brownian motion effects significant in nanofluid heat transfer enhancement?." International Journal of Thermal Sciences 57 (2012): 152-162. https://doi.org/10.1016/j.ijthermalsci.2012.01.016

[15] Sharma, Jyoti, Urvashi Gupta, and Veena Sharma. "Modified model for binary nanofluid convection with initial constant nanoparticle volume fraction." Journal of Applied Fluid Mechanics (JAFM) 10, no. 5 (2017): 1387-1395. https://doi.org/10.18869/acadpub.jafm.73.242.27754

[16] Menni, Younes, Ali J. Chamkha, and Houari Ameur. "Advances of nanofluids in heat exchangers -A review." Heat Transfer 49, no. 8 (2020): 4321-4349. https://doi.org/10.1002/htj.21829

[17] Gupta, Urvashi, Jyoti Sharma, and Mamta Devi. "Casson nanofluid convection in an internally heated layer." Materials Today: Proceedings 28 (2020): 1748-1752. https://doi.org/10.1016/j.matpr.2020.05.156 
[18] Gupta, Urvashi, Jyoti Sharma, and Mamta Devi. "Double-diffusive instability of Casson nanofluids with numerical investigations for blood-based fluid." The European Physical Journal Special Topics (2021): 1-11. https://doi.org/10.1140/epjs/s11734-021-00053-9

[19] Aliouane, Imane, Noureddine Kaid, Houari Ameur, and Houssem Laidoudi. "Investigation of the flow and thermal fields in square enclosures: Rayleigh-Bénard's instabilities of nanofluids." Thermal Science and Engineering Progress 25 (2021): 100959. https://doi.org/10.1016/i.tsep.2021.100959

[20] Mahammedi, Abdelkader, Houari Ameur, Younes Menni, and Driss Meddah Medjahed. "Numerical study of turbulent flows and convective heat transfer of Al2O3-water nanofluids in a circular tube." Journal of Advanced Research in Fluid Mechanics and Thermal Sciences 77, no. 2 (2021): 1-12. https://doi.org/10.37934/arfmts.77.2.112

[21] Douha, Mohammed, Draoui Belkacem, Kaid Noureddine, Ameur Houari, Belkacem Abdellah, Mohamed Elmir, Merabti Abdelhak, and Aissani Houcine. "Study of Laminar Naturel Convection in Partially Porous Cavity in the Presence of Nanofluids." Journal of Advanced Research in Fluid Mechanics and Thermal Sciences 79, no. 1 (2021): 91-110. https://doi.org/10.37934/arfmts.79.1.91110

[22] Abdelkader, Mahammedi, Houari Ameur, and Younes Menni. "Investigation of the convective heat transfer and friction factor of magnetic $\mathrm{Ni}$ nanofluids within cylindrical pipes." transfer $2 \quad$ (2021): 4. https://doi.org/10.36963/IJTST.2021080101

[23] Sparrow, Ephraim M., Richard J. Goldstein, and V. K. Jonsson. "Thermal instability in a horizontal fluid layer: effect of boundary conditions and non-linear temperature profile." Journal of Fluid Mechanics 18, no. 4 (1964): 513-528. https://doi.org/10.1017/S0022112064000386

[24] Char, Ming-I., and Ko-Ta Chiang. "Stability analysis of Benard-Marangoni convection in fluids with internal heat generation." Journal of Physics D: Applied Physics 27, no. 4 (1994): 748. https://doi.org/10.1088/0022$3727 / 27 / 4 / 012$

[25] Nield, D. A., and Andrey V. Kuznetsov. "Thermal instability in a porous medium layer saturated by a nanofluid." International Journal of Heat and Mass Transfer 52, no. 25-26 (2009): 5796-5801. https://doi.org/10.1016/j.ijheatmasstransfer.2009.07.023

[26] Nanjundappa, C. E., I. S. Shivakumara, Jinho Lee, and M. Ravisha. "Effect of internal heat generation on the onset of Brinkman-Bénard convection in a ferrofluid saturated porous layer." International journal of thermal sciences 50, no. 2 (2011): 160-168. https://doi.org/10.1016/i.ijthermalsci.2010.10.003

[27] Yadav, Dhananjay, R. Bhargava, and G. S. Agrawal. "Boundary and internal heat source effects on the onset of Darcy-Brinkman convection in a porous layer saturated by nanofluid." International Journal of Thermal Sciences 60 (2012): 244-254. https://doi.org/10.1016/j.ijthermalsci.2012.05.011

[28] Shivakumara, I. S., and M. Dhananjaya. "Penetrative Brinkman convection in an anisotropic porous layer saturated by a nanofluid." Ain Shams engineering journal 6, no. 2 (2015): $703-713$. https://doi.org/10.1016/j.asej.2014.12.005

[29] Chand, R., G. C. Rana, and S. Kumar. "Variable gravity effects on thermal instability of nanofluid in anisotropic porous medium." International Journal of Applied Mechanics and Engineering 18, no. 3 (2013). https://doi.org/10.2478/ijame-2013-0038

[30] Hamid, Nur Zarifah Abdul, Nor Fadzillah Mohd Mokhtar, Norihan Md Arifin, and Mohammad Hasan Abdul Sathar. "Effect of Nonlinear Temperature Profile on Thermal Convection in a Binary Fluid Saturated an Anisotropic Porous Medium." Journal of Advanced Research in Fluid Mechanics and Thermal Sciences 56, no. 1 (2019): 43-58.

[31] Rusdi, Nadia Diana Mohd, Nor Fadzillah Mohd Mokhtar, Norazak Senu, and Siti Suzilliana Putri Mohamed Isa. "Effect of Coriolis force and magnetic field on thermal convection in an anisotropic porous medium." Journal of Advanced Research in Fluid Mechanics and Thermal Sciences 56, no. 1 (2019): 20-30.

[32] Rusdi, Nadia Diana Mohd, Nor Fadzillah Mohd Mokhtar, Norazak Senu, and Siti Suzilliana Putri Mohamed Isa. "Stability Convection in a Couple Stress Fluid Saturated in an Anisotropic Porous Medium with Internal Heating Effect." Journal of Advanced Research in Fluid Mechanics and Thermal Sciences 76, no. 2 (2020): 75-84. https://doi.org/10.37934/arfmts.76.2.7584

[33] Senin, Nor Halawati, Nor Fadzillah Mohd Mokhtar, and Mohamad Hasan Abdul Sathar. "Ferroconvection in an Anisotropic Porous Medium with Variable Gravity." Journal of Advanced Research in Fluid Mechanics and Thermal Sciences 71, no. 2 (2020): 56-68. https://doi.org/10.37934/arfmts.71.2.5668

[34] Wang, Yuzhou, Jonathan Singer, and Haim H. Bau. "Controlling chaos in a thermal convection loop." Journal of Fluid Mechanics 237 (1992): 479-498. https://doi.org/10.1017/S0022112092003501

[35] Tang, Jie, and Haim H. Bau. "Feedback control stabilization of the no-motion state of a fluid confined in a horizontal porous layer heated from below." Journal of Fluid Mechanics 257 (1993): $485-505$. https://doi.org/10.1017/S0022112093003179

[36] Bau, Haim H. "Control of Marangoni-Bénard convection." International Journal of Heat and Mass Transfer 42, no. 7 (1999): 1327-1341. https://doi.org/10.1016/S0017-9310(98)00234-8 
[37] Hashim, I., and Z. Siri. "Stabilization of steady and oscillatory Marangoni instability in rotating fluid layer by feedback control strategy." Numerical Heat Transfer, Part A: Applications 54, no. 6 (2008): 647-663. https://doi.org/10.1080/10407780802289384

[38] Roslan, R., M. N. Mahmud, and I. Hashim. "Effects of feedback control on chaotic convection in fluid-saturated porous media." International journal of heat and mass transfer 54, no. 1-3 (2011): $404-412$. https://doi.org/10.1016/j.ijheatmasstransfer.2010.09.031

[39] Mahmud, M. N., and I. Hashim. "Small and moderate Vadasz number chaotic convection in porous media in the presence of non-Boussinesq effects and feedback control." Physics Letters A 375, no. 24 (2011): 2382-2393. https://doi.org/10.1016/i.physleta.2011.05.024

[40] Vadasz, Peter. "Coriolis effect on gravity-driven convection in a rotating porous layer heated from below." Journal of Fluid Mechanics 376 (1998): 351-375. https://doi.org/10.1017/S0022112098002961

[41] Govender, Saneshan. "Coriolis effect on the stability of centrifugally driven convection in a rotating anisotropic porous layer subjected to gravity." Transport in porous media 67, no. 2 (2007): $219-227$. https://doi.org/10.1007/s11242-006-9003-5

[42] Khalid, Izzati Khalidah, Nor Fadzillah Mohd Mokhtar, Zarina Bibi Ibrahim, and Zailan Siri. "Rayleigh-Bénard convection in Maxwell nanofluids layer saturated in a rotating porous medium with feedback control subjected to viscosity and thermal conductivity variations." Applied Nanoscience 10, no. 8 (2020): 3085-3095. https://doi.org/10.1007/s13204-020-01302-4

[43] Sharma, Jyoti, and Urvashi Gupta. "Double-diffusive nanofluid convection in porous medium with rotation: DarcyBrinkman model." Procedia Engineering 127 (2015): 783-790. https://doi.org/10.1016/i.proeng.2015.11.413

[44] Sharma, Jyoti, Urvashi Gupta, and R. K. Wanchoo. "Numerical study on binary nanofluid convection in a rotating porous layer." Differential Equations and Dynamical Systems 25, no. 2 (2017): $239-249$. https://doi.org/10.1007/s12591-015-0268-4 